\title{
Perturbation treatment of boundary conditions for fluxon motion in long Josephson junctions
}

Olsen, O. H.; Pedersen, Niels Falsig; Samuelsen, Mogens Rugholm; Svensmark, Henrik; Welner, D.

Published in:

Physical Review B

Link to article, DOI:

10.1103/PhysRevB.33.168

Publication date:

1986

Document Version

Publisher's PDF, also known as Version of record

Link back to DTU Orbit

Citation (APA):

Olsen, O. H., Pedersen, N. F., Samuelsen, M. R., Svensmark, H., \& Welner, D. (1986). Perturbation treatment of boundary conditions for fluxon motion in long Josephson junctions. Physical Review B, 33(1), 168-173.

https://doi.org/10.1103/PhysRevB.33.168

\section{General rights}

Copyright and moral rights for the publications made accessible in the public portal are retained by the authors and/or other copyright owners and it is a condition of accessing publications that users recognise and abide by the legal requirements associated with these rights.

- Users may download and print one copy of any publication from the public portal for the purpose of private study or research.

- You may not further distribute the material or use it for any profit-making activity or commercial gain

- You may freely distribute the URL identifying the publication in the public portal 


\title{
Perturbation treatment of boundary conditions for fluxon motion in long Josephson junctions
}

\author{
O. H. Olsen, * N. F. Pedersen, M. R. Samuelsen, H. Svensmark, and D. Welner \\ Physics Laboratory I, The Technical University of Denmark, DK-2800 Lyngby, Denmark
}

(Received 10 June 1985)

\begin{abstract}
The sine-Gordon equation governing the motion of fluxons in the long Josephson junction is investigated by transforming it into a relativistic-particle equation of motion and using a perturbational approach. The effects of a finite junction length, an external magnetic field, as well as the effects of fluxon-antifluxon collisions are included in the calculations. All theoretical results are compared to numerical simulations in order to investigate the validity of the approach. Good agreement is found in most cases.
\end{abstract}

\section{INTRODUCTION}

Recently there has been an increased interest in soliton propagation in various physical systems. One of the systems where soliton propagation is most accessible for experimental measurements is the long Josephson junction, as is well described in the recent book by Barone and $\mathrm{Pa}$ terno. ${ }^{1}$ Further reviews of the topic have been given in Refs. 2 and 3 . In the Josephson junction the physical manifestation of a soliton is a fluxon, i.e., a quantum of magnetic flux $\Phi=h / 2 e$. The simplest and most direct way of measuring moving fluxons in the long Josephson junction is to measure the so-called zero-field steps in the dc $I V$ curve. The first zero-field step (ZFS) is explained as a resonant motion of a single fluxon along the junction. ${ }^{4}$ Higher-order steps are numbered according to the total number of fluxons and antifluxons. This motion is, in general, governed by a perturbed sine-Gordon ${ }^{5}$ equation subject to boundary conditions determined by such factors as the geometry, the current distribution, ${ }^{6}$ the loading, and the external magnetic field. ${ }^{7}$ If the junction is sufficiently long the size of the fluxon can be neglected. The dynamics of the fluxon is then governed by a relativistic particle equation of motion that may be derived from the sineGordon equation. In that case the driving forces are determined by the current. ${ }^{8}$ The boundary conditions affect the fluxon's particle motion in three ways. ${ }^{9}$ Primarily, the reflections at the ends determine the fluxon frequency and therefore (through the Josephson frequencyto-voltage relation) the voltage. Secondly, this voltage is modified by reflection losses and energy inputs and outputs due to applied magnetic fields. Thirdly, a phase shift due to the mutual attraction of a fluxon and a (virtual) antifluxon gives a further correction to the voltage.

It is the purpose of this paper to derive closed analytical expressions (based on a perturbation treatment) for the first ZFS with different current injections, and to compare these results with corresponding full numerical simulations of the basic perturbed sine-Gordon equation. We also treat the case of externally applied magnetic fields and compare those results to a numerical solution of an equation such as the relativistic particle equation of motion. For both types of comparison, excellent agreement is achieved.
This paper is organized in the following manner: Sec. II describes the model and the basic equations. In Sec. III perturbational methods are applied to those equations. Section IV and subsections discuss the various geometries and finally Sec. V contains the conclusion.

\section{THE MODEL}

The dynamics of a long Josephson tunnel junction is assumed to be governed by a perturbed sine-Gordon equation $^{5}$

$$
\phi_{x x}-\phi_{t t}=\sin \phi+\alpha \phi_{t}+\eta(x),
$$

where $\phi$ is the phase difference between the two superconducting films. The spatial variable is measured in units of the Josephson penetration depth $\lambda_{J}=\left(\hbar / 2 e d \mu_{0} J\right)^{1 / 2}$, and the time in units of the reciprocal plasma frequency $\omega_{0}^{-1}$, where $\omega_{0}=(2 e J / \hbar C)^{1 / 2}$. Here, $J$ is the Josephson current density, $d$ is the magnetic thickness of the barrier, and $C$ is the capacitance per unit area. The loss parameter $\alpha$ is defined through the relation $\alpha=G(\hbar / 2 e J C)^{1 / 2}$, where $G$ is the shunt conductance per unit area. $\eta(x)$ is the normalized density of the current distribution along the Josephson junction, i.e., $\eta(x)=I(x) / W J$, where the width $W$ satisfies the relation $W \ll \lambda_{J} . \quad \eta(x)$ depends on the geometry and the details of the bias current injected into the junction.

With an applied magnetic field $H_{e}$ perpendicular to the length of the junction and parallel to the plane of the barrier and/or with a current injection through the ends of the junction, the boundary conditions for the phase difference $\phi$ at the ends $(x= \pm l / 2)$ are ${ }^{10}$

$$
\pm \phi_{x}\left[\mp \frac{l}{2}, t\right]=\kappa_{1,2}=\kappa \pm \kappa_{\text {ext }} \text {. }
$$

$\kappa$ is the normalized current input through the ends and $\kappa_{\text {ext }}$ is the normalized magnetic field $\kappa_{\text {ext }}=H_{e} / J \lambda_{J} . \quad l$ is the normalized length of the junction $\left(l=L / \lambda_{J}\right)$. Accordingly, the total current $I$ through the junction is given by

$$
I=J W \lambda_{J}\left(2 \kappa+\int_{-l / 2}^{l / 2} \eta(x) d x\right) \equiv J W L \eta_{I}
$$


In this equation the parameter $\eta_{I}$ is the normalized total current.

The problem considered here can be reformulated because part of the boundary condition Eq. (2), can be transformed into $\delta$-function singularities in $\eta(x)$ at the ends (or vice versa). ${ }^{11,12}$ For instance, Eq. (2) could be replaced by

$$
\phi_{x}\left( \pm \frac{l}{2}, t\right)=0
$$

if $\eta(x)$ in Eq. (1) is replaced by $\eta^{\prime}(x)$ given by

$$
\begin{aligned}
\eta^{\prime}(x) & =\eta(x)+2 \kappa_{1} \delta\left(x+\frac{l}{2}\right)+2 \kappa_{2} \delta\left(x-\frac{l}{2}\right) \\
& =\eta(x)+2\left(\kappa \pm \kappa_{\text {ext }}\right) \delta\left(x \pm \frac{l}{2}\right) .
\end{aligned}
$$

$\eta$ and $\eta^{\prime}$ differ only by the singularities at the ends of the junction.

\section{PERTURBATION APPROACH}

The relativistic equation of motion for the fluxonwhich is a $2 \pi$ kink in the phase difference $\phi$-is most easily derived from the soliton momentum $p$ (Refs. 8 and 13),

$$
p=-\frac{1}{8} \int \phi_{x} \phi_{t} d x
$$

which for the pure sine-Gordon equation is given by ${ }^{5}$

$$
p=u \gamma(u) \text {, }
$$

where $u$ is the normalized velocity and $\gamma(u)=\left(1-u^{2}\right)^{-1 / 2}$. The standard normalizations used here may be found in Refs. 2, 3, and 5. By differentiating Eq. (6) with respect to time and using Eq. (1) one obtains the equation of motion for a $\pm 2 \pi$ kink,

$$
\frac{d p}{d t}=-\alpha p \pm \frac{\pi}{4} \eta(x) \text {. }
$$

Equation (8) is expected to be valid if the spatial variation of $\eta(x)$ is negligible over the width of the $2 \pi$ kink $\left(\sim \lambda_{J}\right)$. The approximation then consists of setting $p$ equal to the pure sine-Gordon value in Eq. (7). Equation (8) is the relativistic equation of motion for a particle with the current acting as the force.

The boundary conditions are treated conveniently by considering the energy $H$,

$$
H=\frac{1}{8} \int\left[\frac{1}{2} \phi_{x}^{2}+\frac{1}{2} \phi_{t}^{2}+(1-\cos \phi)\right] d x,
$$

which for the pure sine-Gordon equation is

$$
H=\gamma(u) \text {. }
$$

Owing to the boundary condition, Eq. (2), an energy increase $\Delta H_{1}$ is obtained when a $2 \pi$ kink at $x=l / 2$ is reflected as a $-2 \pi$ kink (and by symmetry when a $-2 \pi$ kink at $x=-l / 2$ is reflected as a $2 \pi$ kink). $\Delta H_{1}$ is given by ${ }^{10}$

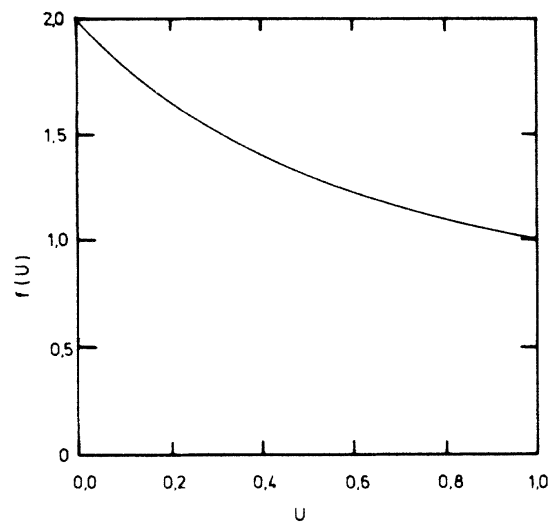

FIG. 1. Function $f(u)$ from Eq. (12) determined numerically.

$$
\Delta H_{1}\left( \pm \frac{l}{2}\right)=\frac{\pi}{2}\left(\kappa \mp \kappa_{\mathrm{ext}}\right) .
$$

Furthermore due to intrinsic losses in the collision process itself, there will be an additional (negative) energy input per reflection, $\Delta H_{2}$, given by ${ }^{9,11}$

$$
\Delta H_{2}=-\frac{\pi^{2} \alpha}{4} f(u) \text {. }
$$

The function $f(u)$ is shown in Fig. 1. This latter energy contribution may formally be included in Eq. (11) by replacing $\kappa$ with $\kappa^{\prime}=\kappa-\pi \alpha f(u) / 2$. Since $u$ is normally close to 1 we will use $f(u)=1$ in the remainder of this work.

The last effect of the boundary is the phase shift due to fluxon-antifluxon attraction., ${ }^{9}$, This phase shift may be accounted for mathematically by decreasing the normalized length of the junction with an amount $\delta$, given by

$$
\delta=-2\left(1-u^{2}\right)^{1 / 2} \ln u .
$$

Thus, to include all boundary corrections, one first calculates an average velocity $u_{\text {av }}^{\prime}$ from Eqs. (8) and (7) by using the energy inputs, Eqs. (11) and (12), at the ends. Subsequently, the phase shift corrected average velocity $u_{\text {av }}$ is obtained from

$$
u_{\mathrm{av}}=u_{\mathrm{av}}^{\prime}\left(1-\frac{\delta}{l}\right)^{-1},
$$

where $\delta$ is given by Eq. (13) with $u=u_{\text {av }}^{\prime}$.

\section{FIRST ZERO-FIELD STEP}

From the perturbation theory developed in the preceding section we now calculate the first zero-field step for different current injections and with an applied magnetic field. Common for all of the cases treated is that $\eta(x)$ is a constant $(=\eta)$. The momentum of the fluxon, $p$, then relaxes exponentially towards the power-balance value $p=\pi \eta / 4 \alpha$. 


\section{A. In-line junction}

In this case, all of the current is fed through the ends of the junction [ $\eta(x)=0$ ] making an exact solution with the perturbation approach possible. The only change from a previous treatment of this problem ${ }^{10}$ is to include the reflection losses to find $u_{\mathrm{av}}^{\prime}$ [see Eqs. (11) and (12)]. $u_{\mathrm{av}}^{\prime}$ is determined by

$$
\kappa-\frac{\pi \alpha}{2} f(u)=\frac{\frac{4}{\pi} \sinh \left(\frac{\alpha l}{2}\right)}{\left[\left[\frac{\tanh \left(\frac{\alpha l}{2 u_{\mathrm{av}}^{\prime}}\right)}{\tanh \left(\frac{\alpha l}{2}\right)}-1\right]^{2}\right]^{1 / 2}}
$$

Equation (15) is only a translation of $\pi \alpha f(u) / 2$ of the results of Ref. 10 along the $\kappa$ axis (the current axis). The phase shift corrected average velocity $u_{\text {av }}$ is then found from E. (14). If the fluxon velocities in the two ends differ too much Eq. (13) should be replaced by ${ }^{11}$

$$
\delta=-\left(1-u_{1}^{2}\right)^{1 / 2} \ln \left(u_{1}\right)-\left(1-u_{2}^{2}\right)^{1 / 2} \ln \left(u_{2}\right),
$$

where $u_{1}$ and $u_{2}$ are the velocities in the two ends (or equivalently before and after the reflection). They are determined by

$$
u_{1,2}=\tanh \left(a_{0} \pm \frac{\alpha l}{2}\right) \text {, }
$$

where $a_{0}$ is found from

$$
\tanh \left(a_{0}+\frac{\alpha l}{2}\right)=\tanh \left(\frac{\alpha l}{2}\right) / \tanh \left(\frac{\alpha l}{2 u_{\mathrm{av}}^{\prime}}\right) .
$$

In Fig. 2 we show the result of the perturbation calculation for the current $\kappa$ versus the voltage $u_{\mathrm{av}}^{\prime}$ with $\alpha=0.1$

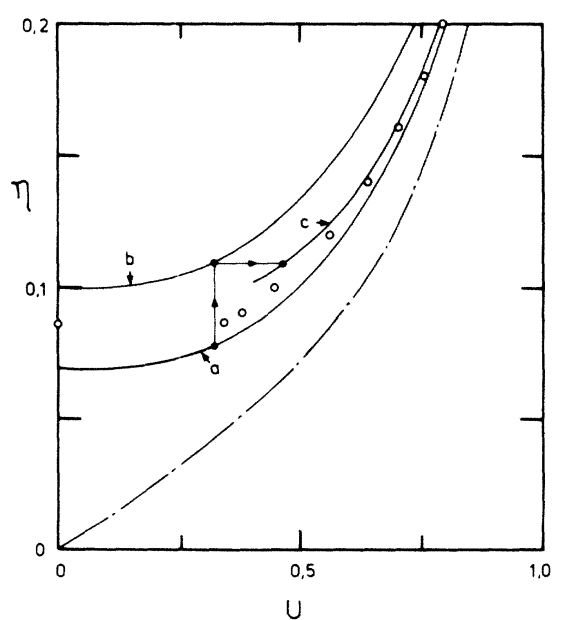

FIG. 2. $I V$ characteristic of an in-line junction with $\alpha=0.1$ and $l=10$. (a) Equation (15) with $f(u)=0$. (b) Includes the reflection loss [Eq. (15)] and (c) includes the reflection loss and the phase shift, Eqs. (16) and (14). Circles are full numerical results. The dashed curve shows the overlap case without reflection loss [Eq. (25)]. and $l=10$. The two corrections [Eqs. (15) and (14)] are shown explicitly. For comparison the result of a full numerical solution of Eqs. (1) and (2) is also shown. Except for the lowest current values the agreement between the perturbation calculation and the full numerical simulation is excellent. With an externally applied magnetic field we may also solve the problem analytically for the first ZFS. In that case the starting point is the analytical solution, Eq. (28) of Ref. 10, without reflection loss and phase shift; then the translation $\kappa \rightarrow \kappa^{\prime}=\kappa-\pi \alpha f(u) / 2$ (reflection loss) is made and finally Eqs. (14) and (15) are used (phase shift correction). Since the analytical expression is rather large, and since no numerical simulations exist for comparison, we do not present the result here.

\section{B. Homogeneous overlap}

In this case all of the current is uniformly distributed and $\eta(x)$ is constant. The reflection loss has the same effect as a negative current input through the ends as can be seen from Eqs. (11) and (12). Thus, we can simultaneously treat the reflection loss and some in-line contribution to the current. However, it must be remembered that the negative contribution from the reflection loss does not contribute to the current.

The perturbation problem considered is equivalent to a relativistic particle moving with damping in a periodic force field with the spatial period $l$ (with magnetic field the period is $2 l$ ). The periodic force is given from Eq. (8) with the periodic continuation of Eq. (5). [The $\kappa$ in Eq. (5) should be substituted with $\kappa^{\prime}$ to include the reflection losses.]

Below we will show that for a given amount of work per period the average velocity $u_{\mathrm{av}}^{\prime}$ will be maximum if $\eta(x)$ is constant, $\kappa=\kappa_{\text {ext }}=0$ and the reflection losses are neglected. Hence, all perturbations will lower the average velocity. We note from Eqs. (7) and (10) that

$$
\frac{d p}{d t}=\frac{d H}{d x} \text {. }
$$

When the fluxon motion becomes stationary (periodic), $\Delta H$ per period is zero. Including the singularities at the ends we may obtain from Eqs. (8) and (19) the general expression

$$
\int_{-l / 2}^{l / 2} p d x=\int_{-l / 2}^{l / 2} \frac{\pi \eta^{\prime}(x)}{4 \alpha} d x \equiv \eta^{\prime} l \frac{\pi}{4 \alpha}=p l .
$$

[with an external magnetic field the spatial period is doubled and $l$ should be replaced by $2 l$ everywhere in Eq. (20) and in what follows]. The temporal period is

$$
T \equiv \frac{l}{u_{\mathrm{av}}^{\prime}}=\int_{-l / 2}^{l / 2} \frac{d x}{u}
$$

If we now assume a constant $\eta^{\prime}(x)=\eta$ and make a change in $\eta^{\prime}(x)$ by an amount $\Delta \eta(x)$, such that $\Delta \eta(x)$ adds no extra work, i.e.,

$$
\int_{-l / 2}^{l / 2} \Delta \eta(x) d x=0
$$

then Eq. (21) can be written as follows: 


$$
\begin{aligned}
T & =\frac{l}{u_{\mathrm{av}}^{\prime}} \\
& \cong \int_{-l / 2}^{l / 2}\left[\frac{1}{u}+\frac{\partial}{\partial p}\left[\frac{1}{u}\right] \Delta p+\frac{1}{2} \frac{\partial^{2}}{\partial p^{2}}\left[\frac{1}{u}\right](\Delta p)^{2}\right] d x \\
& =\frac{l}{u}+\frac{1}{2} \frac{\partial^{2}}{\partial p^{2}}\left[\frac{1}{u}\right] \int_{-l / 2}^{l / 2}(\Delta p)^{2} d x,
\end{aligned}
$$

since from Eq. (22) $\int \Delta p d x$ is zero. Because $p=u \gamma(u)$ we have

$$
\frac{\partial^{2}}{\partial p^{2}}\left(\frac{1}{u}\right)=\frac{\left(2+u^{2}\right)\left(1-u^{2}\right)^{2}}{u^{3}}>0, \text { if } u>0
$$

showing that any change of $\eta^{\prime}(x)=\eta$ fulfilling Eq. (22) yields an increase in $T$ (decrease in $u_{\text {av }}^{\prime}$ ) which is quadratic in the change in $\eta^{\prime}(x)$. This proves the statement made in the beginning of this section that the time period, $T$, is minimum in the absence of perturbations. Thus, the velocity $u$ determined from

$$
p=u \gamma(u)=\frac{\pi \eta}{4 \alpha}
$$

is the maximum velocity obtainable from any current distribution $\eta^{\prime}(x)$ for which

$$
\frac{1}{l} \int_{-l / 2}^{l / 2} \eta^{\prime}(x) d x=\eta
$$

and this maximum is obtained only if $\eta^{\prime}(x)$ is constant and thus equal to $\eta$.

On the basis of the considerations above it is easy to derive analytically the $I V$ curve of the homogeneous overlap junction taking the reflection loss into account. This was done in Ref. 9 and will not be repeated here.

\section{Overlap with some in-line character and magnetic field}

The technique used in Sec. IV B to show that a constant bias yield the largest average velocity for a given average "force" [Eqs. (23) and (24)] will now be used to derive perturbation expressions for $u_{\mathrm{av}}^{\prime}$ in the most general case with some in-line current feed, reflection losses, and an external magnetic field.

The problem is now reduced to determining $\Delta p$ as a function of $x$ in the stationary case with energy inputs, Eqs. (11) and (12), at the singularities. The time period $T$ is then calculated from Eq. (23). From Eq. (8) it follows that the momentum $p$ relaxes exponentially towards the equilibrium value $\pi \eta / 4 \alpha$; however, with the results of the preceding section in mind the deviation $\Delta p$ should be measured relative to the average momentum $p_{0}=\pi \eta_{0} / 4 \alpha$, where $\eta_{0}$ is defined by

$$
\eta_{0}=\eta_{I}-\frac{\pi \alpha}{l} f(u), \quad \eta_{I}=\eta+\frac{2 \kappa}{l}
$$

The time variable in the solution $\Delta p(t)$ is eliminated by setting $t=x / u_{0}$, where $u_{0}$ and $p_{0}$ are related through Eq. (25).

The result of this rather lengthy calculation is

$$
\begin{aligned}
\frac{1}{u_{\mathrm{av}}^{\prime}}= & \frac{1}{u_{0}}+\frac{1}{2} \frac{\left(2+u_{0}^{2}\right)\left(1-u_{0}^{2}\right)^{2}}{u_{0}^{3}} \\
\times & \left\{\left(\frac{\pi \kappa_{\mathrm{ext}}}{2 \alpha l}\right]^{2} \frac{\alpha l}{2 u_{0}} \tanh \left[\frac{\alpha l}{2 u_{0}}\right]\right. \\
& \left.+\left(\frac{\pi \kappa^{\prime}}{2 \alpha l}\right]^{2}\left[\frac{\alpha l}{2 u_{0}} \operatorname{coth}\left[\frac{\alpha l}{2 u_{0}}\right]-1\right]\right\}
\end{aligned}
$$

with

$$
\kappa^{\prime}=\kappa-\frac{\pi \alpha}{2} f(u) .
$$

The first term in Eq. (28) is the same as the magnetic tuning term in Eq. (19) of Ref. 10. The second term in Eq. (28) is almost the same as Eq. (14) of Ref. 9. The difference-apart from the fact that Eq. (28) is more general-is that in Ref. 9 the expansion was carried out around $\eta_{I}$ where we here use $\eta_{0}$. The latter gives better results in a comparison with numerical solutions.

In Fig. 3 the result of the perturbation calculation Eq. (28) is shown together with a numerical solution of the equation of motion for $\kappa_{\text {ext }}=0$. Reflection losses are assumed absent $[f(u)=0]$ but an in-line current component, described by the parameter $y$, is taken into account. $\alpha=0.1$ and $l=10$. We show the average velocity $u_{\text {av }}^{\prime}$, with the average force $\eta_{0}$ as a parameter versus $y$. The quantity $y$ is the fraction of the total current which is fed homogeneously into the junction. ${ }^{6}$ i.e.,

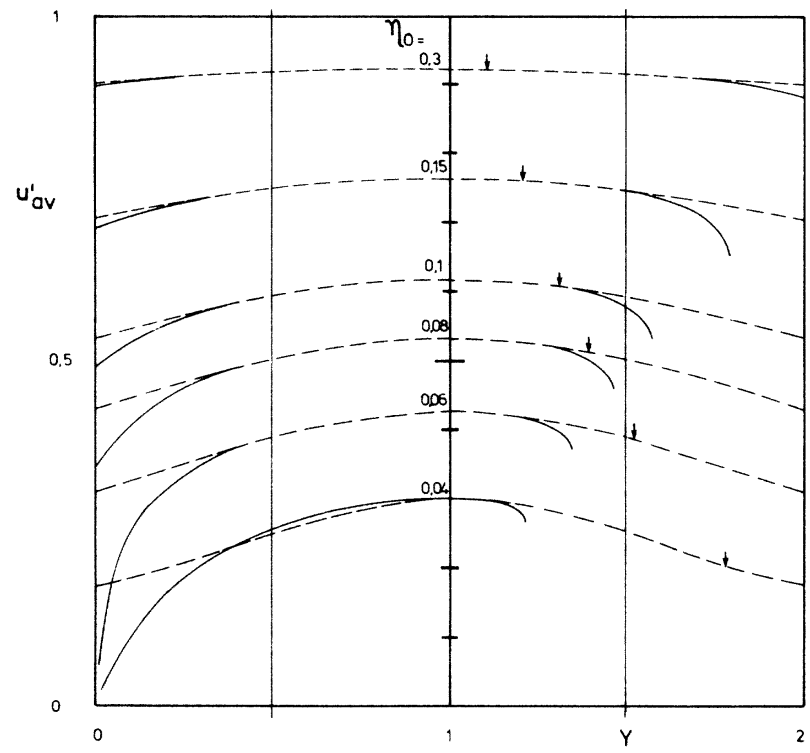

FIG. 3. Dashed curve is the average velocity $u_{\mathrm{av}}^{\prime}$ from Eq. (28) vs $y$, with $\alpha=0.1, l=10, f(u)=0$ (no reflection loss) and $\kappa_{\mathrm{ext}}=0 . y=\eta l / 2\left(\kappa^{\prime}+\eta l\right)$ is the fraction of the current which is spatially uniform. Thus, $y=0$ correspond to the in-line case and $y=1$ to the overlap case. The solid curve is a numerical solution of the same problem. The parameter is the total bias $\eta_{0}$. 


$$
y=\frac{\eta l}{2\left(\kappa^{\prime}+\eta l\right)} .
$$

Consequently $(1-y)$ is the fraction of the total current in the spikes at the ends. The dotted curves in Fig. 3 are the results of the perturbation calculation and the full curve is obtained from a numerical solution. We note the good agreement especially for larger currents $\eta_{0}$. Also we note that $u_{\text {av }}^{\prime}$ is rather insensitive to the way in which the current is fed into the junction. The inclusion of reflection losses can be seen also from Fig. 3, because it is equivalent to a negative in-line current contribution $(y>1)$. For instance, the result for the homogeneous overlap junction with reflection losses is obtained from Fig. 3 by making the following changes [see Eq. (27) with $f(u)=1]$ :

$$
y=1+\frac{\pi \alpha}{l \eta_{0}}
$$

and

$$
\eta_{I}=\eta_{0}+\frac{\pi \alpha}{l}
$$

The $y$ values corresponding to this are shown by the arrows in Fig. 3. The numerical part of the solution (solid curve) also shows the annihilation of the fluxons. ( $u_{\mathrm{av}}^{\prime}$ goes to zero abruptly in the right side of the diagram.)

The effect of a magnetic field is demonstrated in Fig. 4. This figure shows for $\alpha=0.1$ and $l=10$ both the perturbation calculation and the numerical solution for the

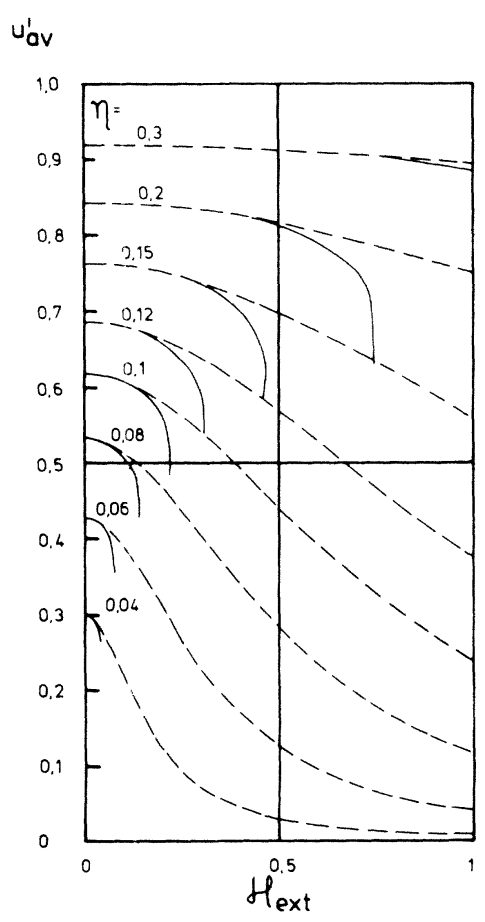

FIG. 4. Overlap junction: The dashed curve is the average velocity $u_{\text {av }}^{\prime}$ from Eq. (28) vs $\kappa_{\text {ext }}$ with $\alpha=0.1, l=10, f(u)=0$ (no reflection losses), and $\kappa=0$. The solid curve is a numerical solution of the same problem. The parameter is the total bias $\eta_{0}$.

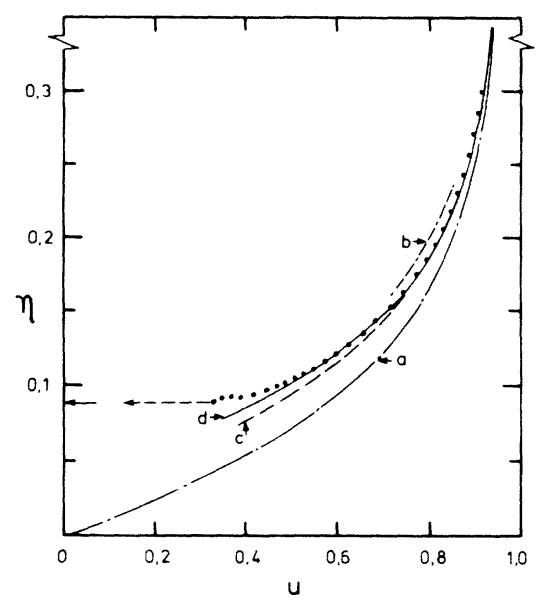

FIG. 5. IV characteristic of a overlap junction with $\alpha=0.1$ and $l=10$. (a) is Eq. (25), (b) includes the reflection loss, Eqs. (27), (28), and (29) with $f(u)=1$. (c) includes reflection loss $[f(u)=1]$ and phase shift Eq. (14). (d) includes velocitydependent reflection loss and phase shift. Circles are full numerical solutions of Eq. (1).

homogeneous overlap case and external magnetic field. $\kappa=0$ and $f(u)=0$ (no reflection losses). Again good agreement is observed and the insensitivity of $u_{\text {av }}^{\prime}$ to the actual shape of the average force is noted. The terminations corresponding to fluxon annihilation are observed as in Fig. 3.

Common to Figs. 3 and 4 is the insensitivity of $u_{\mathrm{av}}^{\prime}$ to the shape of the force. Thus, the main effect of the reflection loss is the translation along the current axis [Eqs. (27) and (29)]. The second-order correction may be found from Figs. 3 and 4 or calculated directly from Eq. (28).

Eventually when $u_{\text {av }}^{\prime}$ has been determined, Eqs. (14) and (13) are used to include the phase shift correction. In Fig. 5 we show the result of this procedure for a homogeneous overlap junction with reflection losses together with the result of a full numerical solution of Eqs. (1) and (2). The agreement is seen to be good.

\section{SUMMARY AND CONCLUSION}

It was demonstrated in this paper that the use of the perturbation approach is feasible for a number of problems relating to soliton propagation on the Josephson transmission line. Thus, the finite length, different geometries, collisions, and magnetic fields could be taken into account, all at the same time. The dynamics could be understood from a simple model of a relativistic particle. However, there are still a number of interesting unsolved problems for which the perturbation theory may give the answers. Some of the unsolved problems that deserve attention are (i) qualitative behavior at boundary conditions with $\phi_{x} \neq 0$, (ii) a calculation of the height of zero-field steps, (iii) bunched fluxon propagation, (iv) the effect of an applied microwave field, and (v) complicated behavior in a large magnetic field. We hope to deal with some of those problems in a future publication. 
*Permanent address: Systems Engineering, NIRO Atomizer, Gladsaxevej 305, DK-2800 Soeborg, Denmark.

${ }^{1}$ A. Barone and G. Paterno, Physics and Applications of the Josephson Effect (Wiley, New York, 1982).

${ }^{2}$ R. D. Parmentier, in Solitons in Action, edited by K. Lonngren and A. C. Scott (Academic, New York, 1978), p. 173.

${ }^{3}$ N. F. Pedersen, in Advances in Superconductivity, edited by B. Deaver and John Ruvalds (Plenum, New York, 1982), p. 149.

${ }^{4}$ T. A. Fulton and R. C. Dynes, Solid State Commun 12, 57 (1973).

${ }^{5}$ D. W. McLaughlin and A. C. Scott, Phys. Rev. A 18, 1652 (1978).

${ }^{6}$ O. H. Olsen and M. R. Samuelsen, J. Appl. Phys. 54, 6522 (1983).
${ }^{7}$ O. H. Olsen and M. R. Samuelsen, J. Appl. Phys. 52, 6247 (1981).

${ }^{8}$ O. H. Olsen and M. R. Samuelsen, Phys. Rev. B 28, 210 (1983). ${ }^{9}$ N. F. Pedersen, M. R. Samuelsen, and D. Welner, Phys. Rev. B 30, 4057 (1984).

${ }^{10}$ O. A. Levring, N. F. Pedersen, and M. R. Samuelsen, J. Appl. Phys. 54, 987 (1983).

${ }^{11}$ P. Bodin, N. F. Pedersen, M. R. Samuelsen, and D. Welner, IEEE Trans. Magn. MAG-21 (to be published).

${ }^{12}$ M. R. Samuelsen and S. A. Vasenko, J. Appl. Phys. 57, 110 (1985).

${ }^{13}$ O. A. Levring, M. R. Samuelsen, and O. H. Olsen, Physica 11D, 349 (1984). 